Boletín de la Sociedad Geológica Mexicana

Volumen 63, NÚM. 1, 2011, P. 83-94

\title{
Evaluación de imágenes del sensor MODIS para la cartografía de la cobertura del suelo en una región altamente diversa de México
}

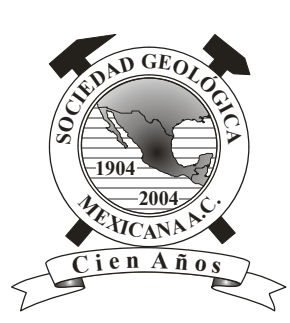

\author{
Tzitziki Janik García-Mora*, Jean-François Mas
}

Centro de Investigaciones en Geografía Ambiental, Universidad Nacional Autónoma de México, Antigua

Carretera a Pátzcuaro No. 8701, Col. Ex-Hacienda de San José de La Huerta, Morelia, 58190, Mich., México

*biol_tzitziki@yahoo.com

\section{Resumen}

En décadas recientes, las actividades agropecuarias se han expandido ocupando cerca del $40 \%$ de la cobertura mundial del suelo impactando gravemente la biodiversidad. Para entender y manejar estos cambios es indispensable contar con datos actualizados sobre el uso y la cobertura del suelo, generados mediante herramientas que permitan obtener información con mayor frecuencia temporal y extensión espacial. Las imágenes derivadas de sensores tales como MODIS representan una alternativa; sin que hasta el momento se cuente con datos precisos de su fiabilidad a nivel regional. Este trabajo pretende contribuir en el conocimiento sobre el tipo de datos más idóneo para generar información de cobertura de suelo, y los métodos que permiten obtener más detalle conservando una fiabilidad aceptable. El área de estudio es la región del Tancítaro, Michoacán y comprende bosques templados y tropicales secos, pastizales y áreas de cultivos. Los tres tipos de datos MODIS; índices de vegetación, compuestos espectrales de 8 días e imágenes de reflectancia diarias fueron evaluados por medio de dos metodologías; la máxima verosimilitud y redes neuronales, en cada una de estas se incorporaron dos tipos de datos auxiliares. Los resultados muestran que es posible obtener mapas confiables a partir de estos datos de baja resolución si se usan categorías generales.

Palabras clave: Datos MODIS, imágenes multiespectrales, índices de vegetación, reflectancia de la superficie, Tancítaro

\begin{abstract}
In recent decades, the use of arable land for agriculture has expanded to occupy nearly $40 \%$ of the world's land surface, thereby greatly impacting the biodiversity of our planet. In order to understand and manage these changes, it is indispensable to have updates on land use/land cover generated with tools that allow us to obtain information over larger areas with greater frequency. Images derived from moderate resolution sensors such as MODIS represent an alternative to high resolution imagery, though we lack a precise understanding of the accuracy of the land characterization provided by this sensor at regional levels. The aim of this work is to contribute to the knowledge about the most ideal type of remote sensing data needed to generate land cover/land use information and the methodologies that can produce a more detailed legend while still retaining an acceptable level of accuracy. The study area is the region of Tancitaro, Michoacan, Mexico and is represented by temperate and dry tropical forests, pasture lands and croplands. Three kinds of MODIS data were tested: vegetation indices, spectral reflectance eight day composites, and daily spectral reflectance images. These data were analyzed through two different approaches; maximum likelihood and neural networks. We also applied ancillary data to compare the results of the classifications with and without the ancillary data. The results obtained show it is possible to achieve acceptable levels of accuracy using moderate resolution imagery if a simple classification scheme is used.
\end{abstract}




\section{Introducción}

La cobertura global del suelo ha sido modificada para proveer de alimento y abrigo a más de seis billones de personas. En décadas recientes, las áreas dedicadas a actividades agropecuarias se han expandido aumentando los requerimientos de energía, agua y fertilizantes ocasionando grandes pérdidas de biodiversidad (Leff et al., 2004; FAO, 2010). Estos cambios en el uso de suelo han permitido al ser humano apropiarse de los recursos, pero al mismo tiempo minan la capacidad de los ecosistemas para sostener la producción de alimento, mantener los recursos forestales, regular el clima, afectan la disponibilidad del agua y la calidad del aire (Foley, 2005). Hoy en día tenemos la tarea de manejar los recursos de manera que sea posible mantener la capacidad de la biosfera para proveer bienes y servicios a largo plazo (Foley, 2005).

Es por lo tanto, indispensable contar con datos actualizados sobre el uso y la cobertura del suelo para entender y manejar estos procesos. Desde la década de los 80 , la percepción remota ha jugado un papel esencial en el monitoreo de las coberturas terrestres. Para estudios locales o regionales, se emplearon ampliamente sensores de mediana resolución espacial (píxeles de unas decenas de metros) como Landsat o SPOT (Sistema Probatorio para la Observación de la Tierra). Para estudios de áreas extensas, se requiere una gran cantidad de imágenes, lo cual vuelve el procesamiento de estos datos largo y costoso. Por ejemplo, se necesitan más de 120 imágenes Landsat para cubrir el territorio mexicano, y el mapeo con base en estas imágenes requiere de una cantidad importante de tiempo y de personal especializado (Mas et al., 2002; Palacio-Prieto et al., 2000). Para estudios a escalas continentales o globales, se utilizaron durante varias décadas imágenes de sensor Advanced Very High Resolution Radiometer (AVHRR). Estas imágenes, diseñadas para la meteorología, presentan algunas limitaciones para estudiar la cobertura terrestre (baja resolución espacial y espectral, saturación de las bandas, mala calidad geométrica). Sin embargo, gracias a su alta resolución temporal (varias tomas al día) permitieron llevar a cabo el monitoreo de la vegetación de manera exitosa en varias partes del mundo incluyendo Norteamérica (Loveland et al., 2000) y Sudamérica (Stone et al., 1994). Recientemente, se lanzaron al espacio sensores de baja resolución espacial y alta resolución temporal especialmente diseñados para el monitoreo de la cobertura terrestre, entre los cuales destacan MODIS y SPOT-Vegetation. MODIS (Moderate Resolution Imaging Spectroradiometer) es un sensor particularmente interesante; tiene 36 bandas espectrales (de las cuales 7 son útiles para estudiar la cobertura terrestre contando con una resolución espacial de 250 y $500 \mathrm{~m}$ ). MODIS está a bordo de dos satélites (Terra y Aqua), permite tener imágenes diarias de toda la superficie terrestre y existen una gran cantidad de productos derivados disponibles en internet.

Existen relativamente pocas aplicaciones de MODIS al mapeo de las coberturas del suelo, en particular en regiones intertropicales con una alta diversidad y complejidad espacial. Este trabajo pretende contribuir a conocer la efectividad de imágenes de baja resolución e índices de vegetación derivados de las mismas bajo diferentes métodos de análisis para evaluar el nivel de discriminación alcanzable, manteniendo una fiabilidad aceptable, en una región con una gran diversidad de tipos de uso/cobertura de suelo, característica común en México y en la mayoría de los países intertropicales.

\section{2. Área de estudio}

El área de estudio cuenta con una superficie de 4,650 $\mathrm{km}^{2}$, se ubica en la región centro-oriental de México (Figura 1), encierra áreas que corresponden a dos provincias fisiográficas del país, la Sierra Madre del Sur y la Faja Volcánica Transmexicana. Cuenta con un amplio rango altitudinal (de 600 a 4,000 m), con climas que varían desde templado-húmedo, semicálido subhúmedo, semicálido húmedo hasta cálido subhúmedo. La temperatura media anual varía desde los $10^{\circ} \mathrm{C}$ en el pico del Tancítaro hasta los $24^{\circ} \mathrm{C}$ en las partes bajas mientras que la precipitación media oscila entre 1,000 a los $1,500 \mathrm{~mm}$. La cobertura vegetal incluye selva baja caducifolia, bosque de pino y de pino-encino además de extensas áreas de agricultura de temporal, agricultura de riego, pastizales y huertas de aguacate. Hay también un área sin vegetación (colada de lava del volcán Paricutín que hizo erupción entre 1943 y 1952) así como ciudades de gran importancia en el estado entre las que destacan Uruapan con 279,229 habitantes y Apatzingán con 114,837 habitantes.

\section{Materiales}

Los insumos se dividen en tres grupos: 1) los datos a evaluar derivados del sensor MODIS, 2) los datos auxiliares utilizados para mejorar las clasificaciones y 3) los datos para medir la fiabilidad de los mapas obtenidos.

Existen diferentes productos obtenidos a partir de las imágenes MODIS. En este estudio utilizamos algunos de los productos enfocados al estudio de las cubiertas terrestres (MODLAND) obtenidos con el satélite Terra. Una revisión de los productos MODLAND puede encontrarse en Mas y García-Mora (en prensa). El producto MOD09 indica la reflectancia (relación entre el flujo luminoso reflejado y el flujo luminoso incidente) para las siete primeras bandas del sensor en el visible y el infrarrojo (Figura 2) y se encuentra disponible en varias resoluciones temporales. Los compuestos de 8 días consisten en combinar las "mejores" observaciones obtenidas durante ocho días para generar una imagen. Los índices de vegetación (producto MOD13) son transformaciones matemáticas de las bandas espectrales originalmente captadas por el sensor remoto. Ejemplos de estos son: el índice de vegetación de diferen- 


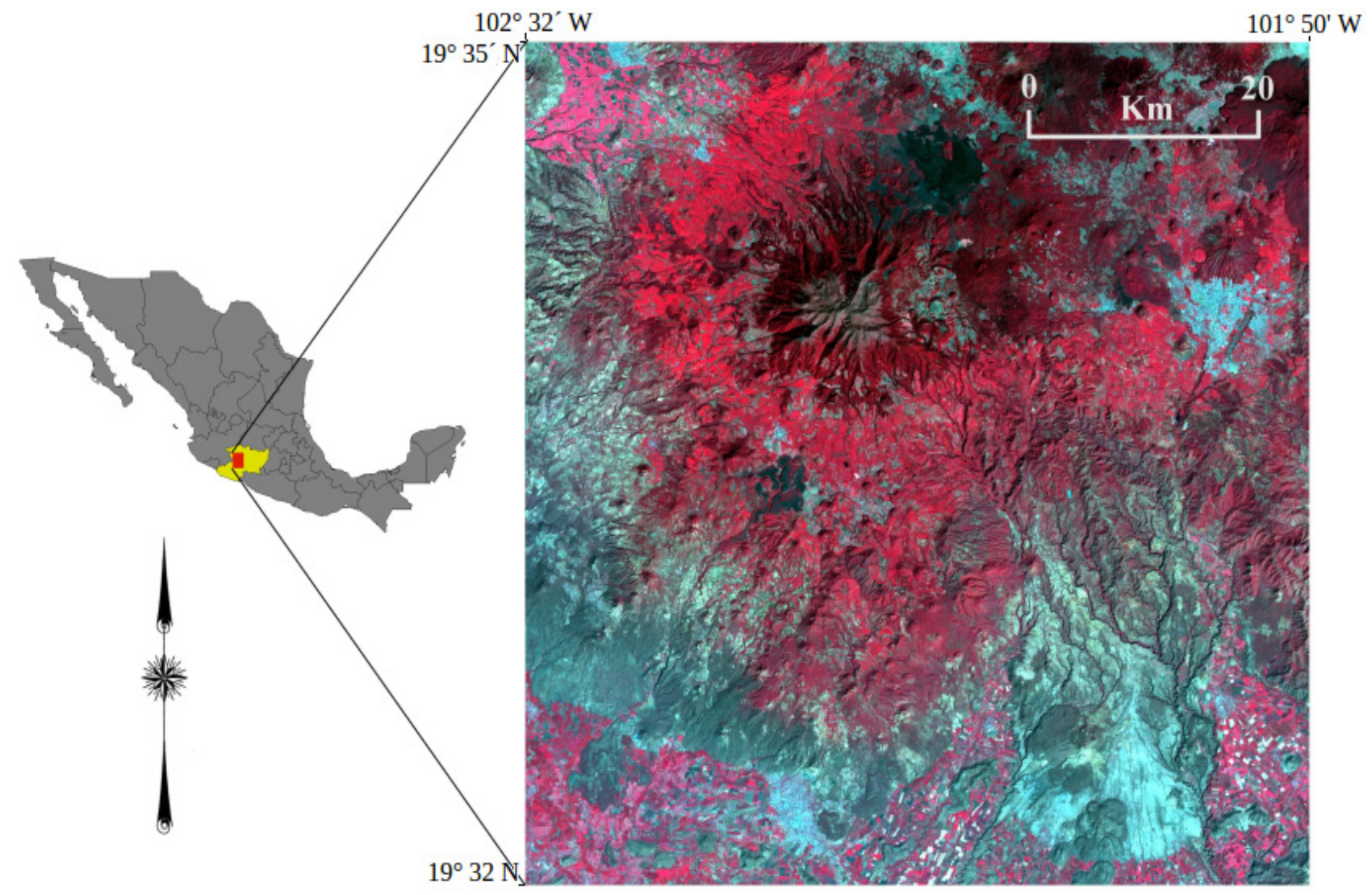

Figura 1. - Localización del área de estudio.

cia normalizada (NDVI) y el índice de vegetación mejorado (EVI). El índice de diferencia normalizada se produce mediante el cálculo del cociente normalizado de la banda del infrarrojo cercano y la banda del rojo.

$N D V I=\frac{R_{I R C}-R_{R}}{R_{I R C}+R_{R}}=\frac{B_{2}-B_{1}}{B_{2}+B_{1}}$

(Ecuación 1)

Donde, $R_{I R C}$ y $R_{R}$ son respectivamente los valores de reflectancia bidireccional de la superficie para las bandas del infrarrojo cercano y rojo, que son las bandas $\mathrm{B}_{2} \mathrm{y}$ $\mathrm{B}_{1}$ (Tucker, 1979). Este índice da continuidad al NDVI obtenido con base en el sensor AVHRR para extender el registro de datos sobre periodos más largos en los estudios de monitoreo.

El índice mejorado de vegetación (EVI), se optimizó a partir del NDVI aumentando la sensibilidad en las regiones de alta biomasa y mejoró el monitoreo de la vegetación mediante la disociación de la señal del fondo del dosel y una reducción de la influencia atmosférica. La ecuación utilizada para su obtención se indica a continuación:

$$
\begin{aligned}
& E V I=G \frac{R_{I R C}-R_{R}}{R_{I R C}+C_{1} R_{R}-C_{2} R_{A}+L} \\
& =G \frac{B_{2}-B_{1}}{B_{2}+C_{1} B_{1}-C_{2} B_{3}+L}
\end{aligned}
$$

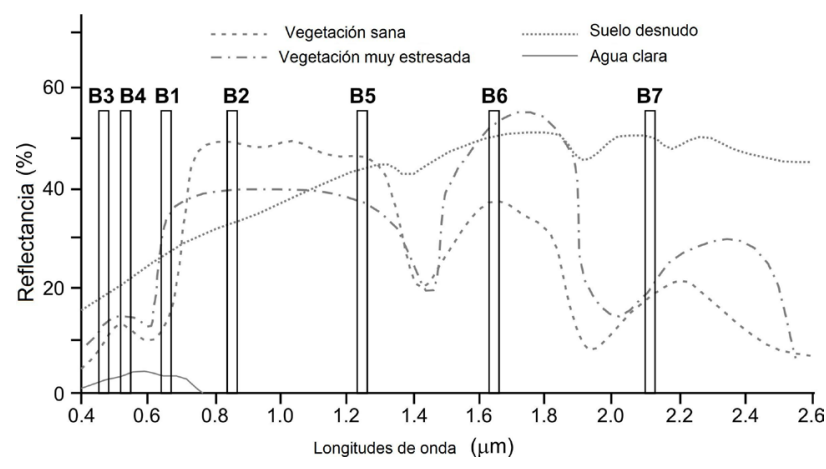

Figura 2. Longitudes de onda de las 7 primeras bandas del sensor MODIS.

Donde:

$R_{I R C}, R_{R}$ y $R_{A}$ son respectivamente los valores de reflectancia bidireccional de la superficie para las bandas del infrarrojo cercano, del rojo y del azul (bandas $\mathrm{B}_{2}, \mathrm{~B}_{1}$ y $\mathrm{B}_{3}$ respectivamente) con una corrección de los efectos de la atmósfera (Absorción por ozono y dispersión de Rayleigh).

$L$ es un ajuste del fondo del dosel que toma en cuenta la transferencia radiante diferencial del infrarrojo cercano y el rojo a través del dosel, $G$ es un factor de ganancia, y $C_{1}, C_{2}$ son los coeficientes de resistencia de aerosoles, que usan la banda azul para corregir la influencia del aerosol en 
la banda roja. Los coeficientes adoptados en el algoritmo EVI son $\mathrm{L}=1, \mathrm{C}_{1}=6, \mathrm{C}_{2}=7.5, \mathrm{y} \mathrm{G}=2.5$ (Liu y Huete, 1995).

Los productos terrestres MODIS utilizados en el presente estudio fueron de dos tipos: 1) el producto de reflectancia de la superficie (MOD09) para el cual se usaron datos compuestos de ocho días (MOD09Q1 y MOD09A1) e imágenes diarias (MOD09GQ y MOD09GA) a una resolución de 250 y $500 \mathrm{~m}$, y 2) el producto de índices de vegetación (MOD13Q1, NDVI) y el índice de vegetación mejorado (EVI) con una resolución espacial de $250 \mathrm{~m}$ en compuestos de 16 días. Los datos se obtuvieron para toda la duración del año 2002 y se componen por lo tanto de 24 composiciones de ambos índices de vegetación de 16 días, 365 imágenes diarias con 7 bandas cada una y 48 compuestos multiespectrales de 8 días también con 7 bandas cada uno. Todos los datos MODIS utilizados fueron obtenidos del satélite Terra, en la última versión disponible (colección 5) de manera gratuita por medio del portal Warehouse Inventory Search Tool (WIST). Cada imagen incluye una capa de información que indica, píxel a píxel, la calidad de los datos.

Los datos auxiliares utilizados fueron los mapas del Inventario Nacional Forestal (IFN) 2000 y de uso del suelo y vegetación del Instituto Nacional de Estadísticas, Geografía e Informática (INEGI, 2002), ambos a escala 1:250,000 así como una imagen Landsat del 2003. Se utilizó también un mapa edafológico escala 1:250000 de INEGI (2000) y un modelo digital de elevación del Shuttle Radar Topography Mission (NASA, 2010a) con $90 \mathrm{~m}$ de resolución (Farr y Kobrick, 2000).

Se llevó a cabo la interpretación de orto-fotografías del INEGI escala 1:20000 del año 2002 y visitas de campo para elaborar una base de datos con 600 puntos de verificación, la cual se utilizó para evaluar la fiabilidad de los mapas obtenidos con MODIS.

El procesamiento de los datos MODIS se llevó a cabo utilizando los programas MODIS Reprojection Tool (MRT) y Land Data Operational Product Evaluation (LDOPE) de acceso libre. Las clasificaciones se hicieron con el programa IDRISI.

\section{Métodos}

El análisis de los datos MODIS se llevó a cabo siguiéndose tres pasos principales 1) el preprocesamiento que consistió en seleccionar las "mejores" bandas de entrada, 2) la clasificación de los datos seleccionados por dos métodos, incluyendo el uso de datos auxiliares y 3) la evaluación de la fiabilidad (Figura 3).

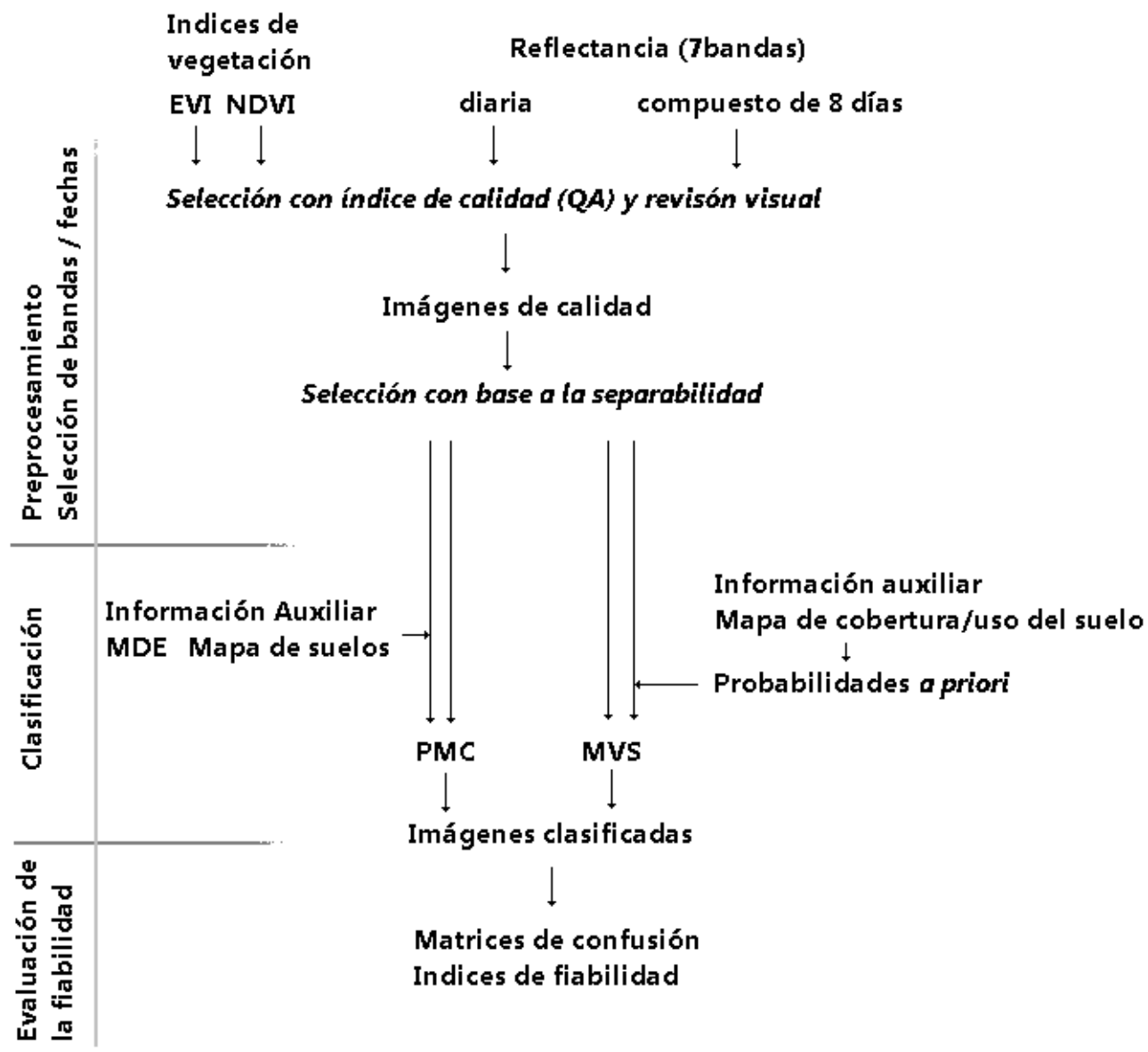

Figura 3. Diagrama de flujo describiendo el procedimiento de análisis de los datos MODIS. 


\subsection{Preprocesamiento}

Los productos MODIS se recortaron y reproyectaron de su proyección original sinusoidal a la proyección Universal Transversal de Mercator (UTM, zona 14). Se evaluó el índice de utilidad de la capa de calidad para descartar las imágenes de baja calidad. En los índices de vegetación, este índice está codificado en los bits 2-5 y varía de 0 (más alta calidad) a 7 (imagen inutilizable). En los productos de reflectancia se encuentra en los bits $0-1$ y varía de 0 (corregido a calidad ideal en todas las bandas) a 3 (no producido). Además en las imágenes diarias en los bits 2-3 se encuentra codificada la información sobre el estado de nebulosidad con los valores de 0 (píxel sin nube), 1 (píxel con mezcla de nube) y 2 (píxel con nube). Una descripción completa de los índices de calidad se encuentra en NASA (2010b). Con base en el índice de utilidad se seleccionaron aquellos compuestos de reflectancia de 8 días e índices de vegetación que presentaron la más alta calidad en todos sus píxeles (imágenes cuyo índice de utilidad promedio es igual a 0). En las imágenes diarias se descartaron las escenas que presentaron $10 \%$ o más de píxeles con nube o bien, que tuvieron píxeles con un valor mayor a 0 en el índice de calidad. Se revisaron visualmente las imágenes seleccionadas.

Se elaboró un sistema clasificatorio con clases de cobertura basadas en las categorías de clasificación del INEGI (2007) y del IFN 2000. Para la zona de estudio, las categorías son:

1) bosque de pino-encino, 2) bosque de pino, 3) selva baja caducifolia, 4) agricultura de riego, 5) huertas de aguacate, 6) agricultura de temporal, 7) pastizales, 8) áreas sin vegetación y 9) áreas urbanas. Este sistema es detallado comparado con los que se usan generalmente en este tipo de imágenes, como el sistema clasificatorio del International Geosphere-Biosphere Programme o IGBP (Belward et al., 1999; Running et al., 1995).

Se elaboraron campos de entrenamiento con la ayuda de la imagen Landsat, las orto-fotografías y la cartografía disponible. Generalmente, el desempeño del clasificador aumenta al aumentar el número de bandas de entrada. Sin embargo, a partir de un cierto número de bandas, el desempeño ya no aumenta, e inclusive disminuye. Este efecto de saturación, conocido como el efecto Hughes, afecta a los clasificadores de máxima verosimilitud y a las redes neuronales. Para identificar el número óptimo de bandas y el conjunto de bandas más eficiente para diferenciar las categorías que se pretenden cartografiar, se puede evaluar la separabilidad entre pares de categorías a través del cálculo de la distancia entre las muestras (campo de entrenamiento) en el espacio multidimensional definido por un cierto conjunto de variables de entrada. Este enfoque permite identificar que categorías se confunden entre sí (aquellas que presentan poca separabilidad) y toma en cuenta los valores de separabilidad de todos los pares de categorías, para definir la combinación de bandas de entrada que permite la mejor separabilidad general (Landgrebe, 2003). Con el objeto de reducir la dimensionalidad de la base de datos se evaluó la separabilidad, a través del cálculo de la divergencia transformada (DT) porque permite prever el desempeño del clasificador de máxima verosimilitud (Maussel et al., 1990), indicando cuantas y cuales bandas son suficientes para discriminar las clases de interés. Los valores de la DT fluctúan entre 0 y 2000 , donde el valor de 2000 representa la separabilidad máxima entre los pares de clases. El cálculo de la separabilidad se hizo primero por cada tipo de producto MODIS (compuestos multiespectrales, imágenes diarias e índices de vegetación), y en una segunda fase para combinaciones de productos. Para evaluar cada conjunto de bandas, se tomó en cuenta el valor mínimo de separabilidad, correspondiente al par de categorías que más tienden a confundirse.

\subsection{Clasificación de las imágenes MODIS}

Para conducir las clasificaciones se eligieron dos clasificadores: de máxima verosimilitud (MV) y el perceptrón multicapa (PMC). El clasificador de máxima verosimilitud (o máxima probabilidad) es el algoritmo más comúnmente utilizado en percepción remota, ya que es sencillo de aplicar, así como de interpretar los resultados que arroja, además de considerarse uno de los procedimientos de discriminación más eficientes, siempre y cuando los datos sigan una función de distribución normal (Maselli et al., 1992). En la fase de aprendizaje, el algoritmo elabora el "patrón espectral" de cada categoría con base en la media y de la varianza/covarianza de un conjunto de sitios de entrenamiento localizados en la imagen. En la fase de clasificación, se calcula, para cada píxel, la probabilidad de pertenencia a cada categoría con base en su respuesta espectral (Ecuación 3). El píxel se asigna finalmente a la clase a la cual es más probable que pertenezca de acuerdo a la información espectral.

$P(H \mid E)=\frac{p(E \mid H) p(H)}{p(E)}$

(Ecuación 3)

Donde $P(H \mid E)$ es la probabilidad condicionada de la hipótesis $H$ dado evidencia $E$, es decir la probabilidad que el píxel pertenezca a cierta categoría (hipótesis $H$ ) tomando en cuenta su respuesta espectral (evidencia $E$ ), $p(H)$ es la probabilidad a priori de la hipótesis $H$, es decir la probabilidad que un píxel pertenezca a la categoría considerada en la hipótesis $\mathrm{H}$ sin tomar en cuenta su información espectral.

$p(E)$ es la probabilidad de la evidencia $E, p(E \mid H)$ es la probabilidad condicionada de la evidencia $E$ dada la hipótesis $H$.

En ausencia de información sobre la probabilidad $a$ priori de encontrar cierta categoría en determinado sitio, 
se supone que las probabilidades a priori $p(H)$ son iguales para todas las categorías y en toda la imagen, es decir que si se consideran $n$ categorías, esta probabilidad es igual a 1/n (Ecuación 4).

$P(H \mid E)=\frac{p(E \mid H)(1 / n)}{p(E)}$

(Ecuación 4)

El Perceptrón Multicapa (PMC), es el algoritmo de redes neuronales más utilizado debido a su capacidad de tolerancia ante información incompleta o contaminada con ruido (Mas y Flores, 2008). Consiste en un modelo estadístico no paramétrico de regresión no lineal (Sarle, 1994) y se describe como una red de alimentación hacia adelante compuesta por una capa de unidades (neuronas) de entrada (en este caso una unidad por banda), otra capa de unidades de salida (una unidad para cada categoría) y un número determinado de capas intermedias de unidades de procesamiento, también llamadas capas ocultas porque no tienen conexiones con el exterior. Las neuronas de cada capa están conectadas a todas las unidades de la capa siguiente (Figura 4). Con esta red se pretende establecer una correspondencia entre un conjunto de entrada (respuesta espectral por ejemplo) y un conjunto de salidas deseadas (categorías de cobertura del suelo). Generalmente, el PMC usa una sola capa oculta para dividir completamente el espacio espectral por medio de hiperplanos a lo largo del cual el nivel de activación de las unidades ocultas es constante (Foody, 2000).

En el PMC, la señal que se transmite es un valor numérico. Cada conexión entre dos neuronas está asociada a un peso de ponderación que controla la fuerza de una interconexión. La neurona recibe como señal de entrada la suma de las señales de salida de las neuronas de la capa anterior, ponderada por su respectivo peso. Esta señal de entrada es transformada por una función de activación, generalmente sigmoidal, que permite generar una señal de salida que se transmite a las neuronas de las capas siguientes. En un PMC cuyo objetivo es clasificar, cada neurona de salida está asociada a una categoría, la categoría final corresponde a la neurona que presentan la señal, o valor de activación, más grande. El valor de activación de cada categoría puede interpretarse con un valor de pertenencia difusa ya que estos valores no se pueden considerar como probabilidad en sentido estricto como en el caso del método de la máxima probabilidad.

Al inicio, los pesos de ponderación de cada conexión se determinan de manera aleatoria, y la respuesta del PMC es por lo tanto totalmente errónea. Con base en los datos de entrenamiento, se modifican los pesos para obtener las respuestas correctas. El procedimiento de aprendizaje es

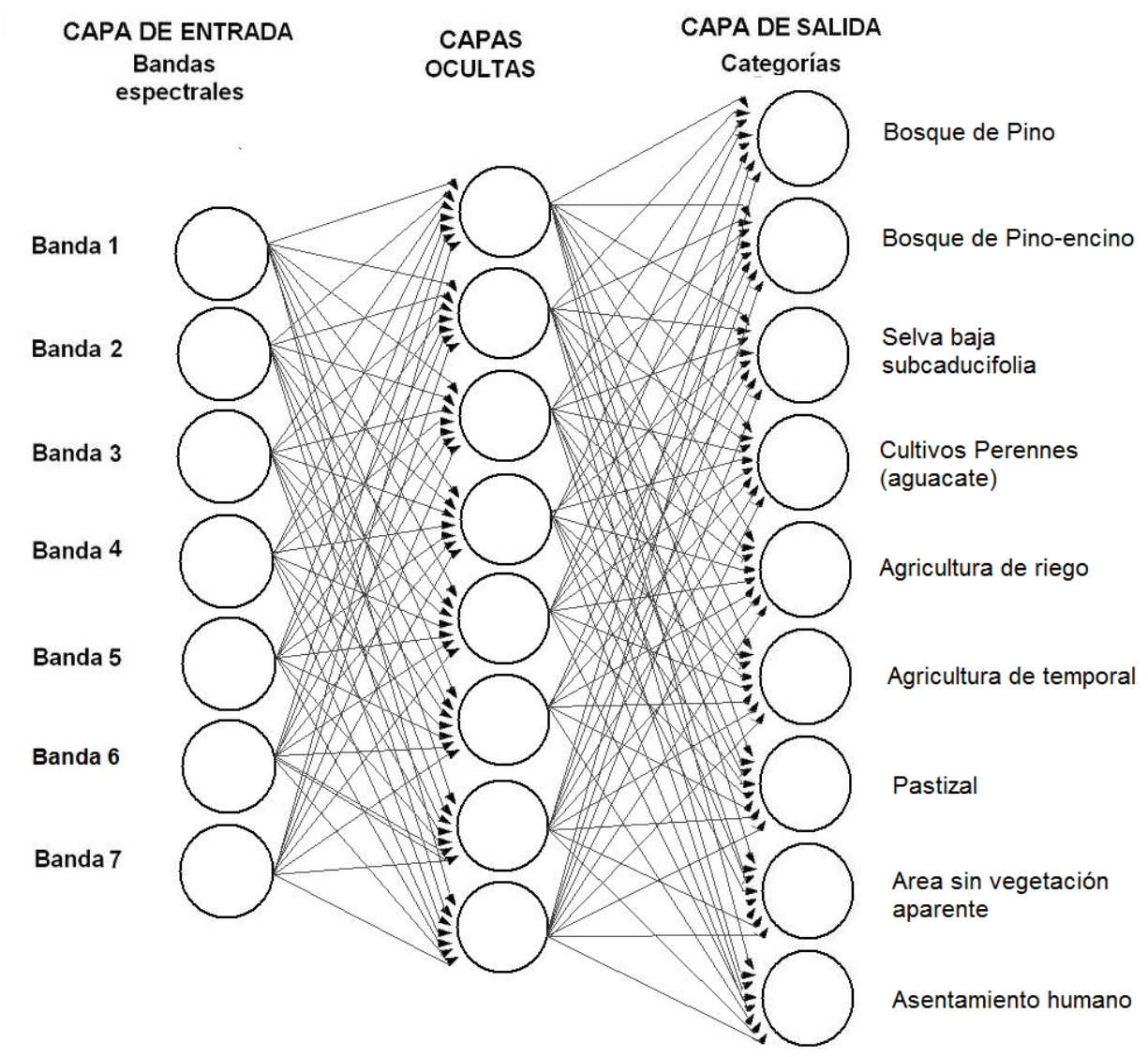

Figura 4. Red Neuronal perceptrón multicapa para clasificar una imagen de siete bandas en nueve categorías. 
sencillo: si la red da la respuesta equivocada, los pesos se corrigen de tal manera que el error disminuye. Los datos de entrenamiento se presentan iterativamente para ajustar los pesos y obtener el mejor ajuste entre valores esperado y obtenido por la red. El método de entrenamiento más conocido es el de retro-propagación (back propagation). En este algoritmo, se compara, con base en los datos de entrenamiento, la señal de salida de la red con el valor esperado para calcular el error. El error es entonces retroalimentado por la red y los pesos de las conexiones se alteran para minimizar este error (Bishop, 1995).

\subsection{Clasificación con información auxiliar}

La incorporación de datos auxiliares en la clasificación ha mostrado aumentar la fiabilidad de los mapas obtenidos (Hutchinson, 1982), por lo que en este estudio se probaron dos formas de incorporar datos auxiliares. El método no paramétrico es ideal para incorporar información no espectral como información auxiliar (Lu y Weng, 2007), mientras que en el método paramétrico se optó por incorporar probabilidades a priori.

Las características del método de máxima verosimilitud lo hacen ideal para incorporar datos auxiliares aumentando su desempeño y conservando las ventajas del método original (Maselli et al., 1995; Wulder et al., 2006). Se generaron mapas de probabilidades a priori $\mathrm{p}(\mathrm{H})$ para cada una de las categorías, aplicando un filtro paso-bajo de $2 \times 2 \mathrm{~km}$ en el mapa de cobertura / uso del suelo de INEGI (2002). En términos prácticos, para cada píxel, estos mapas de probabilidad a priori disminuyen o anulan la probabilidad que el clasificador escoja una categoría que no esté presente en los alrededores del píxel en el mapa de referencia (ver Ecuación 3).

En el caso de las redes neuronales, se usaron los datos de elevación y un mapa de suelos de INEGI (2000), para generar mapas de "probabilidad" a priori (Mas, 2004) que se combinaron con los valores de activación del PMC entrenado con los datos de MODIS. Para ello, el modelo digital de elevación fue clasificado por otro PMC usando el mapa de uso/cobertura del suelo de INEGI (2002) como campos de entrenamiento y reteniendo los valores de activación de la clasificación. Este mapa expresa por lo tanto, para cada píxel, la posibilidad (no se puede hablar de probabilidad en el sentido estricto) de encontrar cada una de las categorías de cobertura tomando en cuenta la elevación. El mismo mapa de INEGI se sobrelapó con el mapa de suelos para calcular la probabilidad condicional de cada categoría de cobertura para cada tipo de suelo. Estos mapas de "posibilidad" se combinaron, categoría por categoría, con el mapa de valores de activación de la clasificación con datos MODIS tomando en cuenta el valor mínimo de los tres valores (derivado de la elevación, del suelo y de los datos MODIS), lo cual corresponde al operador AND en lógica difusa. Finalmente, el píxel se clasificó en la categoría que presentaba el valor máximo de posibilidad final.

\subsection{Análisis de la fiabilidad}

Para evaluar la fiabilidad, se seleccionaron 600 sitios de verificación con base en un muestreo aleatorio estratificado (Stehman y Czaplewski, 1998). La interpretación de los sitios se hizo mediante un enfoque difuso adaptado del propuesto por Woodcock y Gopal (2000). En el caso de que un sitio estuviera situado en el límite entre dos categorías o en un área con clases mezcladas se dio opción para que ambas estuvieran representadas, por lo que para calificar el mapa podía ser considerada como correcta cualquiera de las dos categorías.

Se elaboraron matrices de confusión, las cuales permiten confrontar la información de los sitios de verificación y la imagen clasificada. A esta matriz se aplicó el método propuesto por Card (1982), incorporando los valores de proporción (áreas relativas) de cada una de las categorías consideradas para compensar el sesgo debido al muestreo estratificado.

Se calcularon los valores de la fiabilidad del usuario (proporción de sitios de verificación de la categoría considerada clasificados como tal) y del productor (proporción de sitios clasificado en la categoría considerada en el mapa que están correctamente clasificados). Estos dos índices de fiabilidad están relacionados con los errores de omisión y de comisión respectivamente. Para comparar estadísticamente la fiabilidad de los mapas obtenidos por los diferentes insumos y enfoques de clasificación, se utilizó la prueba de McNemar propuesta por Foody (2004), que permite evaluar la significancia estadística de diferencias en la fiabilidad de dos clasificaciones estimada con base en los mismos datos de verificación. Es una prueba no paramétrica basada en una matriz de confusión de dos por dos que expresa las relaciones entre las confusiones de las dos clasificaciones en comparación (Tabla 1 y Ecuación 5).

Tabla 1. Matriz de confusión utilizada para la prueba de McNemar.

\begin{tabular}{lccc} 
& Clasificación 1 & Correcto & Incorrecto \\
Clasificación 2 & & & \\
\hline Correcto & $f_{11}$ & $f_{21}$ \\
Incorrecto & $f_{12}$ & $f_{22}$ \\
\hline
\end{tabular}

$Z^{2}=\frac{\left(f_{12}-f_{21}\right)}{\left(f_{12}+f_{21}\right)}$

(Ecuación 5)

Donde $f_{11}$ y $f_{22}$ son el número de sitios de verificación correctamente e incorrectamente clasificados en ambas clasificaciones $\mathrm{y}, f_{12}$ y $f_{21}$ son el número de sitios de verificación correctamente clasificados en una de las clasificaciones e incorrectamente clasificados en la otra.

El valor de $Z^{2}$ se compara con el valor crítico de chi cuadrado para un grado de libertad de uno para evaluar si la diferencia de fiabilidad de las dos clasificaciones es significativamente diferente. 


\section{Resultados}

Por medio del análisis de las capas de calidad, se descartaron más de $50 \%$ de los datos. Todas las imágenes descartadas corresponden a la época de lluvias (Figura 5). En el caso de los datos de reflectancia (compuestos de 8 días e imágenes diarias), la información sobre calidad no permitió descartar la totalidad de las imágenes con nebulosidad y se efectuó un análisis visual adicional.

Como se observa en la Figura 6, todas las combinaciones de insumos permiten obtener la separabilidad óptima con un número muy reducido de bandas. Tres compuestos de reflectancia de 8 días permiten obtener una muy alta separabilidad mientras se necesitan 7 índices de vegetación para alcanzar una separabilidad equivalente. Como muestra la Tabla 2, en el caso de los índices de vegetación, el cálculo de la DT indica que las bandas que contribuyen más a separar las categorías son cuatro EVI y tres NDVI, que representan cuatro meses, todos de la época seca.

Dentro de los compuestos de 8 días, el método de separabilidad selecciona como bandas óptimas para discriminar las categorías las correspondientes a las fechas de dos semanas de enero, una de febrero y una de marzo con un abanico espectral amplio (bandas 2, 3, 4, 5 y 6). En las imágenes de reflectancia diarias se seleccionan 2 días de enero y uno de marzo en el rango espectral de las bandas 3, 4, 5 y 6. Para las combinaciones de índices de vegetación con compuestos espectrales de 8 días, se seleccionaron dos EVI, un NDVI y las bandas 1, 3, 6 y 7 de los meses de enero, febrero y diciembre. En la combinación de imágenes diarias con índices de vegetación, resultó más útil la información de reflectancia en el rango de las bandas 1, 2, 4, 5 y 6 y dos EVI que temporalmente abarcan enero, febrero, marzo y mayo.

Precipitación vs. Calidad

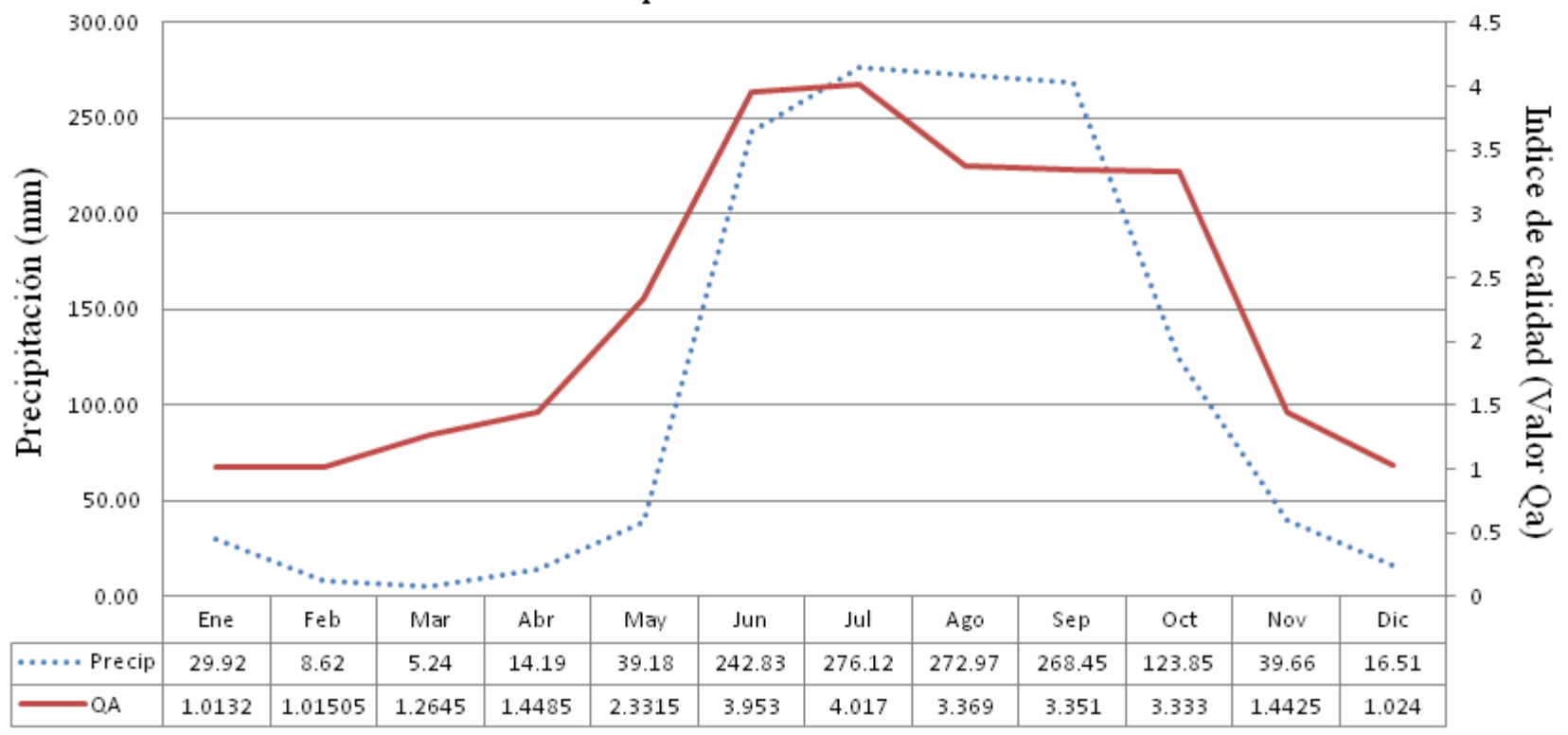

Figura 5. Índice de calidad promedio y precipitación promedio en el área de estudio durante el año 2002 . El índice utilizado (índice de calidad) tiene un valor entre 0 (calidad óptima) y 7 (calidad nula). La precipitación se promedió de 17 estaciones localizadas dentro del área de estudio. Se puede observar la coincidencia entre imágenes de baja calidad y la época de lluvias.

Tabla 2. Bandas y fechas seleccionadas con el criterio de la separabilidad.

\begin{tabular}{|c|c|c|c|c|c|c|c|c|}
\hline \multirow{2}{*}{ IV } & Fecha & 17-ene & 02 -feb & 18-feb & 03-dic & 17-ene & 17-nov & 03-dic \\
\hline & Tipo IV & EVI & EVI & EVI & EVI & NDVI & NDVI & NDVI \\
\hline \multirow{2}{*}{$\mathrm{C} 8$} & Fecha & 09-ene & 09-ene & 25-ene & 25-ene & 10 -feb & 14-mar & 14-mar \\
\hline & Banda & $\mathrm{C} 8 \mathrm{~b} 04$ & $\mathrm{C} 8 \mathrm{~b} 05$ & $\mathrm{C} 8 \mathrm{~b} 05$ & $\mathrm{C} 8 \mathrm{~b} 06$ & $\mathrm{C} 8 \mathrm{~b} 02$ & $\mathrm{C} 8 \mathrm{~b} 03$ & $\mathrm{C} 8 \mathrm{~b} 04$ \\
\hline \multirow{2}{*}{ Diarias } & Fecha & 02-ene & 27-ene & 27-ene & 27-ene & 16-mar & 16-mar & 16-mar \\
\hline & Banda & $\mathrm{db} 06$ & $\mathrm{db} 03$ & $\mathrm{db} 05$ & $\mathrm{db} 06$ & $\mathrm{db} 04$ & $\mathrm{db} 05$ & $\mathrm{db} 06$ \\
\hline \multirow{2}{*}{$\mathrm{IV}+\mathrm{C} 8$} & Fecha & 18-feb & 03-dic & 17-ene & 01-ene & 09-ene & 09-ene & 09-ene \\
\hline & Banda & EVI & EVI & NDVI & $\mathrm{C} 8 \mathrm{~b} 03$ & $\mathrm{C} 8 \mathrm{~b} 01$ & $\mathrm{C} 8 \mathrm{~b} 06$ & $\mathrm{C} 8 \mathrm{~b} 07$ \\
\hline \multirow{2}{*}{ Diarias+IV } & Fecha & 17-ene & 18 -feb & 27-ene & 16-mar & 16-mar & 03-may & 20-nov \\
\hline & Banda & EVI & EVI & $\mathrm{db} 02$ & db04 & db06 & $\mathrm{db} 05$ & $\mathrm{db} 01$ \\
\hline \multirow{2}{*}{$\begin{array}{l}\text { Diarias+IV } \\
+\mathrm{C} 8\end{array}$} & Fecha & 25-ene & 10 -feb & 18 -feb & 14-mar & 14-mar & 09-may & 02 -feb \\
\hline & Banda & C8b06 & $\mathrm{C} 8 \mathrm{~b} 02$ & EVI & $\mathrm{C} 8 \mathrm{~b} 02$ & $\mathrm{C} 8 \mathrm{~b} 04$ & EVI & $\mathrm{db} 06$ \\
\hline
\end{tabular}




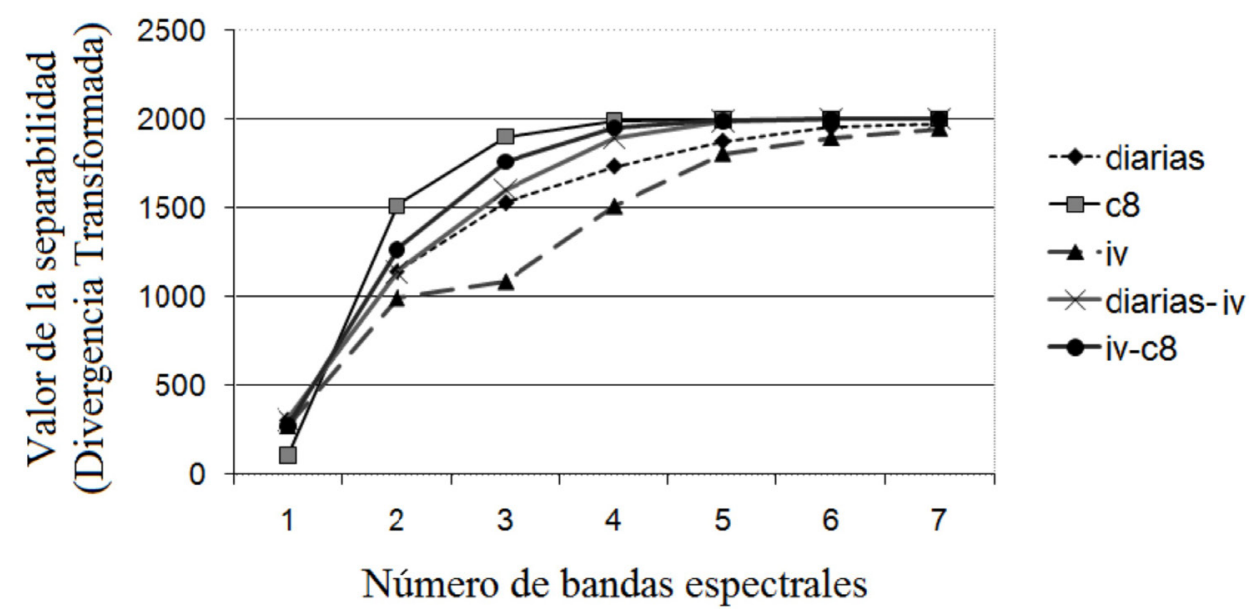

Figura 6. Valor mínimo de divergencia transformada en función del número de bandas de diferentes tipos de productos MODIS (diarias: imágenes de reflectancia diaria, C8: compuestos de reflectancia de 8 días, IV: índices de vegetación).

\subsection{Clasificaciones}

\subsubsection{Máxima verosimilitud (MV) y perceptrón multicapa (PMC)}

La fiabilidad global alcanzada por los diferentes grupos de insumos y métodos de clasificación indican que de manera general, la MV tuvo un mejor desempeño que el PMC (Figura 7). Eso se debe tal vez a la diferencia en el tamaño de los campos (número de píxeles) de entrenamiento de las categorías. Considerando que en la MV, el clasificador utiliza estadísticas derivadas de los campos de entrenamiento, el tamaño del campo no tiene mucha influencia siempre y cuando sea suficientemente grande para tener representatividad estadística. En el caso del PMC, el algoritmo de aprendizaje que busca disminuir el error global tiende a subestimar las categorías poco representadas en los cam- pos de entrenamiento. Con los compuestos de reflectancia de 8 días el clasificador de MV alcanza a clasificar correctamente $74 \%$ de los pixeles, mientras que con los datos combinados (reflectancias diarias, de 8 días e índices de vegetación) se alcanza un $76 \%$, lo cual no representa una diferencia significativa. Este mismo clasificador, utilizando los datos MODIS combinados y adicionalmente datos auxiliares (probabilidades a priori) permite alcanzar una fiabilidad de $83 \%$, esto es significativamente diferente de las clasificaciones que incluyen únicamente datos MODIS.

El método de incorporación de probabilidades a priori derivadas de otro mapa de uso/cobertura del suelo permitió mejorar las clasificaciones más eficientemente que el método basado en el modelo digital de elevación y el mapa de suelo, ya que permitió restringir mucho más las posibilidades de clasificación (Figura 7). Como se puede observar en la Figura 8, muchas categorías tienen una distribución altitudinal muy amplia sobrelapándose entre ellas. De la

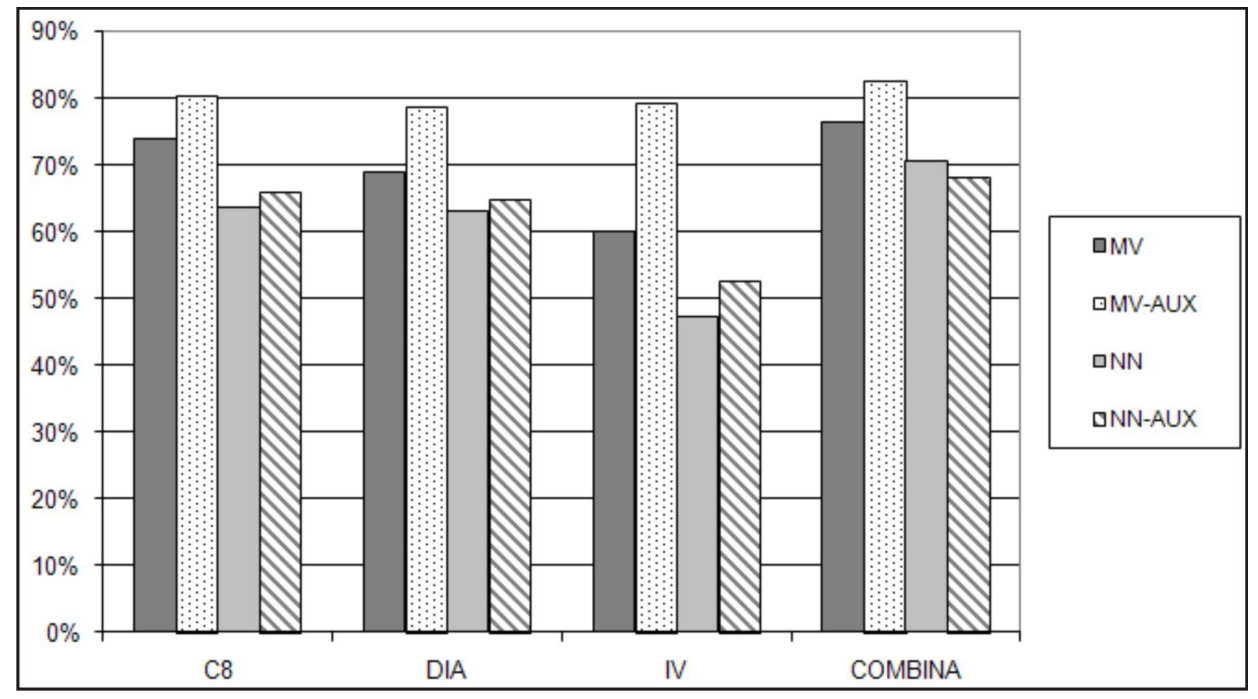

Figura 7. Fiabilidad global alcanzada por los varios métodos/insumos. MV; máxima verosimilitud, AUX; datos auxiliares, PMC; perceptrón multicapa, C8; compuestos de 8 días, DIA; datos diarios, IV; índices de vegetación, COMB; datos combinados. 


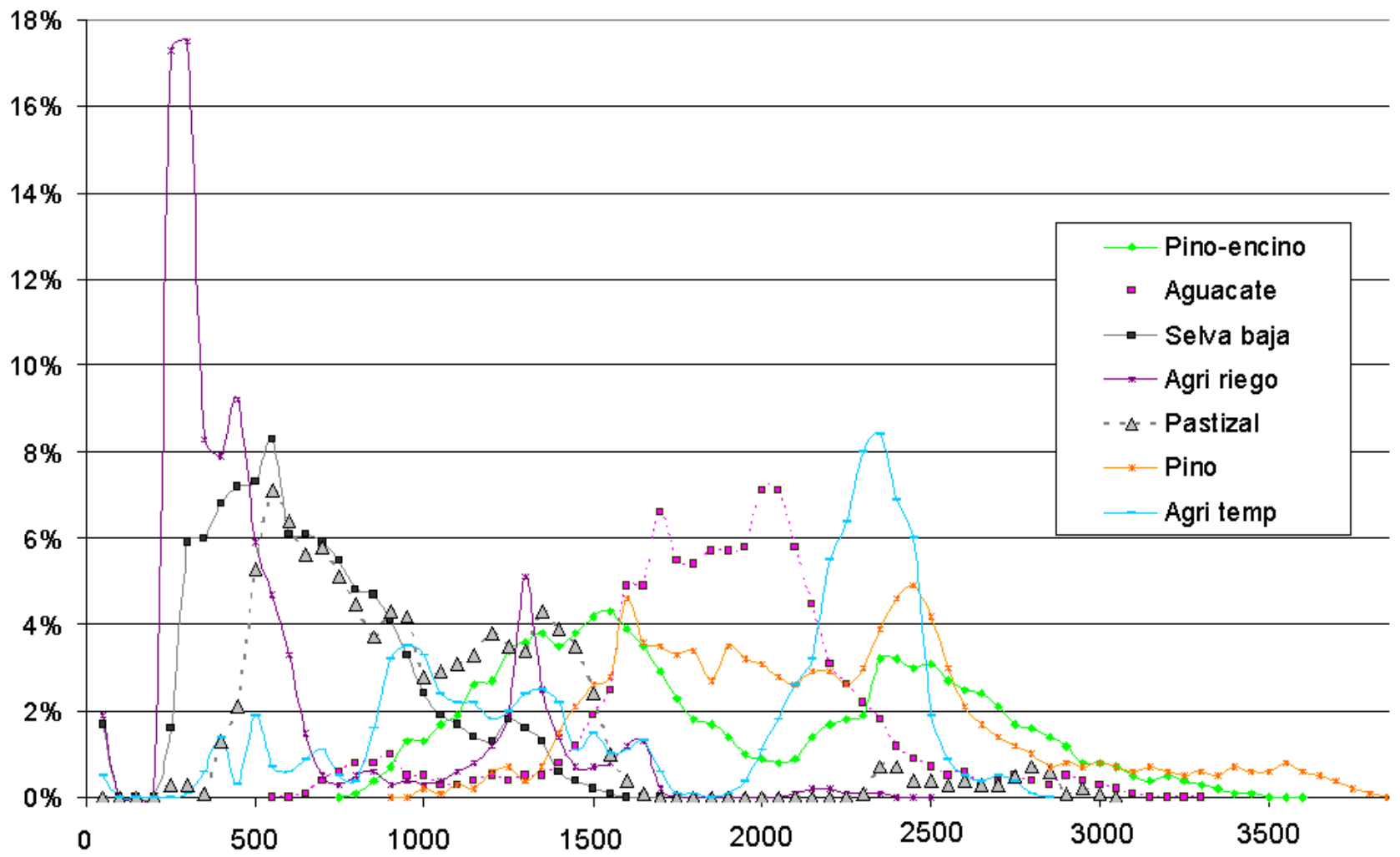

Figura 8. Distribución de diferentes tipos de coberturas (\% del área total de la cobertura) en función de la elevación.

misma manera, los tipos de suelo no corresponden estrictamente con las categorías de cobertura del suelo. Por estas razones, la utilización de este tipo de información auxiliar obtuvo resultados variables. Mientras que en el caso de los datos derivados de índices de vegetación hubo un aumento significativo en la fiabilidad, en el resto de los datos no permitió la obtención de una diferencia significativa.

Como se puede observar en la Tabla 3, todas las categorías tienen una fiabilidad satisfactoria, con rangos de fiabilidad comparables a otros estudios realizados a nivel nacional (Couturier, en prensa; Zhu et al., 2000; Büttner y Maucha 2006) en la clasificación obtenida por el método de máxima verosimilitud con probabilidades a priori derivadas de datos auxiliares.

\section{Discusión y conclusión}

El índice de calidad fue parcialmente útil en la selección de imágenes de buena calidad. Si bien permitió descartar mediante un procedimiento automático las imágenes de mala calidad, fue luego necesaria una selección visual adicional para descartar imágenes de mala calidad que presentaron valores satisfactorios en el índice de calidad.

La selección de las bandas que permiten la mejor separabilidad muestra que la diversidad espectral es más importante que la temporal ya que las bandas seleccionadas corresponden a diferentes partes del espectro pero a fechas cercanas. Carrão et al. (2008) encontraron resultados simi- lares en Portugal, aunque en nuestro caso no hubo casi ninguna imagen de calidad durante todo el periodo de lluvias.

La incorporación de imágenes de probabilidad a priori derivado de un mapa de uso/cobertura existente permitió un incremento significativo de la fiabilidad. Sin embargo, este método podría resultar a veces contraproducente en particular si el mapa de referencia es erróneo o desactualizado, ya que se podrían imponer los errores del mapa al combinar las probabilidades derivadas del análisis de las imágenes de percepción remota y las probabilidades a priori obtenidas del mapa. El tamaño de la ventana del filtro que se utiliza permite determinar el grado en el cual el mapa de referencia se plasma de manera estricta en las probabilidades. En el caso extremo de un filtro de 1 x 1 píxel, los píxeles pertenecientes a un polígono del mapa tendrán una probabilidad uno para la categoría del polígono y cero para las demás, lo cual resultaría en imponer el mapa e ignorar la información de las imágenes. En nuestro estudio, el $38 \%$ de los píxeles tienen una probabilidad superior a cero de pertenecer a 2 categorías, $20 \%$ a tres y el $6 \%$ a 4 ó más. En la práctica, la información de las imágenes de percepción remota puede determinar la categoría en las áreas de contacto entre diferentes categorías pero no en áreas interiores homogéneas en las cuales solo la categoría del mapa de referencia tiene una probabilidad superior a cero.

Tomando en cuenta resultados basados en otro tipo de datos podemos decir que aun sin el uso de información auxiliar, se logró generar un mapa con una fiabilidad sa- 
Tabla 3. Resultados de fiabilidad de usuario y productor (en \%) por categoría de los datos combinados en cada uno de los tipos de clasificación.

\begin{tabular}{lcccccccc}
\hline & \multicolumn{2}{c}{ MV-COMB } & \multicolumn{2}{c}{ MVAUX-COMB } & \multicolumn{2}{c}{ PMC } & \multicolumn{2}{c}{ PMC-AUX } \\
\cline { 2 - 9 } CATEGORIA & CU & CP & CU & CP & CU & CP & CU & CP \\
\hline Pino-encino & 57 & 73 & 76 & 82 & 24 & 32 & 39 & 57 \\
Aguacate & 92 & 81 & 90 & 83 & 89 & 73 & 92 & 69 \\
Sin Veg & 98 & 63 & 96 & 64 & 94 & 64 & 88 & 35 \\
Selva baja & 81 & 84 & 85 & 93 & 86 & 83 & 83 & 83 \\
Agri Riego & 71 & 78 & 86 & 87 & 43 & 69 & 34 & 99 \\
Area urbana & 89 & 72 & 89 & 64 & 88 & 63 & 56 & 100 \\
Pastizal & 89 & 79 & 89 & 74 & 88 & 88 & 56 & 84 \\
Pino & 75 & 77 & 78 & 85 & 47 & 68 & 48 & 69 \\
Agri temp. & 65 & 57 & 73 & 75 & 24 & 33 & 71 & 52 \\
MEDIA & 80 & 74 & 85 & 78 & 65 & 64 & 63 & 72 \\
\hline
\end{tabular}

MV: máxima verosimilitud, AUX: datos auxiliares, PMC: perceptrón multicapa, COMB: datos combinados.

tisfactoria. Por ejemplo, Couturier et al. (2006) evalúan la confiabilidad de una clasificación basada en Landsat en una región cercana en el estado de Michoacán, encontrando una fiabilidad promedio de usuario de $76 \%$ y de productor de $73 \%$, mientras que en este estudio encontramos $80 \%$ y $74 \%$ respectivamente con datos combinados sin el uso de información auxiliar. Vale la pena mencionar que en el caso de la clasificación con datos Landsat obtiene fiabilidades de cero en la categoría de área sin vegetación aparente lo cual reduce mucho la fiabilidad promedio.

Basado en datos MODIS, Carrão et al. (2008) encuentran una fiabilidad media de usuario de $64 \%$ y de productor igual a $46 \%$ tomando en cuenta solo tres clases en su estudio realizado en Portugal, resultados muy por debajo de los obtenidos en este estudio para siete categorías.

En cuanto a otros estudios realizados con datos MODIS, evaluamos la calidad del producto MOD12 que es un mapa de cobertura del suelo basado en el sistema de clasificación del IGBP en la misma área de estudio, los resultados de la comparación de las superficies obtenidas por MOD12 contra la base de datos utilizada para la evaluación de la fiabilidad muestran que este producto presenta errores de hasta el $44 \%$ de la superficie. Sin embargo, se debe considerar que el producto MOD12 se calibra para todo el continente americano.

Los resultados obtenidos nos permiten concluir que los datos MODIS pueden ser utilizados bajo métodos de análisis alternativos, obteniendo resultados suficientemente satisfactorios con base en un sistema clasificatorio bastante detallado para una clasificación obtenida con datos de baja resolución espacial.

\section{Agradecimientos}

Este estudio se llevó a cabo en el ámbito del proyecto 47741 del Fondo Sectorial para la Investigación, el Desarrollo y la Innovación Tecnológica Forestal (CONAFORCONACYT). El mapa de uso del suelo y vegetación (serie III) fue proporcionado por el Instituto Nacional de
Estadística, Geografía e Informática (INEGI) en el ámbito del convenio general de colaboración entre el INEGI y la UNAM.

El primer autor agradece a la beca de doctorado otorgada por CONACYT en la convocatoria 2007. El segundo autor agradece el CONACYT y la DGAPA por el apoyo recibido durante la estancia en la UCSB durante la cual se terminó la elaboración del artículo. Ambos autores agradecen el equipo científico de MODIS por brindar los datos sin restricción, al M. en C. José Antonio Navarrete Pacheco por la elaboración de la Figura 2 y al M. Sc. Everett Hinkley por la edición del resumen en inglés. Los comentarios de tres revisores permitieron mejorar el manuscrito de este artículo.

\section{Referencias}

Belward, A.S., Estes, J.E., Kline, K.D., 1999, The IGBP-DIS Global 1-km Land-Cover Data Set DISCover: A Project Overview: Photogrammetric Engineering \& Remote Sensing, 65, 1013-1020.

Bishop, C.M., 1995, Neural Networks for Pattern Recognition: Nueva York, Oxford University Press, $504 \mathrm{p}$.

Büttner, G., Maucha, G., 2006, The thematic accuracy of CORINE Land Cover 2000: Assessment using LUCAS: Copenhague, Dinamarca, European Environmental Agency, Technical Report No. 7, 85 p.

Card, H.D., 1982, Using known map category marginal frequencies to improve estimates of thematic map accuracy: Photogrammetric Engineering \& Remote Sensing, 48, 431-439.

Carrão, H., Gonçalves, P., Caetano, M., 2008, Contribution of multispectral and multitemporal information from MODIS images to land cover classification: Remote Sensing of Environment, 112, 986-997.

Couturier, S., Mas, J.F., Lopez, E., Cuevas, G., Vega, A., Tapia, V., 2006, Accuracy Assessment Methodology for the Mexican National Forest Inventory: a Pilot Study in the Cuitzeo Lake Watershed (resumen), en 7th International symposium on spatial accuracy assessment in natural resources and environmental sciences: Lisboa, Portugal, Instituto Geografico Portugués, 578-587.

Couturier, S., en prensa, Evaluación de la cobertura vegetal en Mexico: aciertos y sesgos del mapa derivado del sensor MODIS, en Mas, J.F. (coordinador), Aplicaciones del sensor MODIS para el monitoreo del territorio: México, D.F., INE-Semarnat-CIGA-UNAM.

Food and Agriculture Organization of the United Nations (FAO), 2010, Global forest resources assessment 2010: FAO, Roma, Italia, $378 \mathrm{p}$.

Farr, T.G., Kobrick, M., 2000, Shuttle Radar Topography Mission produ- 
ces a wealth of data: Eos, 81, 583-585.

Foley, J.A., DeFries, R., Asner, G.P., Barford, C., Bonan, G., Carpenter, S.R., Chapin, F.S., Coe, M.T., Daily, G.C., Gibbs, H.K., Helkowski, J.H., Holloway, T., Howard, E.A., Kucharik, C.J., Monfreda, C., Patz, J.A., Prentice, I.C., Ramankutty, N., Snyder, P.K., 2005, Global consequences of land use: Science, 309, 570-574.

Foody, G.M., 2000, Mapping land cover from remotely sensed data with a softened feedforward neural network classification: Journal of Intelligent and Robotic Systems, 29, 433-449.

Foody, G.M., 2004, Supervised image classification by MLP and RBF neural networks with and without an exhaustively defined set of classes: International Journal of Remote Sensing, 25, 3091-3104.

Hutchinson, C.F., 1982, Techniques for combining Landsat and ancillary data for digital classification improvement: Photogrammetric Engineering \& Remote Sensing, 48, 123-130.

Inventario Forestal Nacional (IFN), 2000, Mapa de coberturas del suelo y vegetación, Escala 1:250000, Mexico D.F., Conafor-Instituto de Geografía-UNAM-INEGI, 1 mapa.

Instituto Nacional de Estadística, Geografía e Informática (INEGI), 2000, Mapa edafológico, Escala 1:250000: Aguascalientes, Aguascalientes, México, 1 mapa.

Instituto Nacional de Estadística, Geografía e Informática (INEGI), 2002, Mapa de uso del suelo y vegetación, Escala 1:250000: Aguascalientes, Aguascalientes, México, INEGI, 1 mapa.

Instituto Nacional de Estadística, Geografía e Informática (INEGI), 2007, Diccionario de datos de uso del suelo y vegetación 1:250000 (vectorial): Aguascalientes, Aguascalientes, México, INEGI, 50 p.

Leff, B., Ramankutty, N., Foley, J.A., 2004, Geographic distribution of major crops across the world: Global Biogeochemical Cycles, 18, GB1009.

Landgrebe, D.A., 2003, Signal theory methods in multispectral remote sensing: Nueva York, John Wiley and Sons, 528 p.

Liu, H.Q., Huete, A.R., 1995, A feedback based modification of the NDVI to minimize canopy background and atmospheric noise: Institute of Electrical and Electronics Engineers Transactions on Geoscience and Remote Sensing, 33, 457-465.

Loveland, T.R., Reed, B.C., Brown, J.F., Ohlen, D.O., Zhu, Z., Yang, L., Merchant, J.W., 2000, Development of a global land cover characteristics database and IGBP DISCover from $1 \mathrm{~km}$ AVHRR data: International Journal of Remote Sensing, 21, 1303-1330.

Lu, D., Weng, Q., 2007, A survey of image classification methods and techniques for improving classification performance: International Journal of Remote Sensing, 28, 823-870.

Mas, J.F., 2004, Mapping land use/cover in a tropical coastal area using satellite sensor data, GIS and artificial neural networks: Estuarine, Coastal and Shelf Science, 59, 219-230.

Mas, J.F., Flores, J.J., 2008, The application of artificial neural networks to the analysis of remotely sensed data: International Journal of Remote Sensing, 29, 617-663.

Mas, J.F., García-Mora, T., en prensa, MODLAND: Los productos de superficie terrestre MODIS, en Mas, J.F. (coord.), Aplicaciones del sensor MODIS para el monitoreo del territorio: México, D.F., INE-Semarnat-CIGA-UNAM.

Mas, J.F., Velazquez, A., Palacio-Prieto, J.L., Bocco, G., Peralta, A., Prado, J., 2002, Assessing forest resources in Mexico: Wall-towall land use/cover mapping: Photogrammetric Engineering \& Remote Sensing, 68, 966-968.
Maselli, F., Conese, C., Petkov, L., Resti, R., 1992, Inclusion of prior probabilities derived from a nonparametric process into the maximumlikelihood classifier: Photogrammetric Engineering and Remote Sensing, 58, 201-207.

Maselli, F., Conese, C., De Filippis, T., Romani, M., 1995, Integration of ancillary data into a maximum-likelihood classifier with nonparametric priors: ISPRS Journal of Photogrammetry and Remote Sensing, 50, 2-11.

Maussel, P.W., Kramber, W.J., Lee, J.K., 1990, Optimum band selection for supervised classification of multispectral data: Photogrammetric Engineering \& Remote Sensing, 56, 55-60.

National Aeronautics and Space Administration (NASA), 2010a, Shuttle Radar Topographic Mission, Jet Propulsion Laboratory: La Cañada Flintridge, California, EUA, disponible en $<$ http://www2.jpl.nasa. gov/srtm/>, consultado 7 de abril de 2011.

National Aeronautics and Space Administration (NASA), 2010b, MODIS Land Quality Assessment, Goddard Space Flight Center: Greenbelt, Maryland, EUA, disponible en <http://modis-250m.nascom.nasa. gov/cgi-bin/QA_WWW/newPage.cgi?fileName=modland_guide>, consultado $\overline{7}$ de abril de 2011 .

Palacio-Prieto, J.L., Bocco, G., Velazquez, A., Mas, J.F., Takaki Takaki, F., Victoria, A., Luna-Gonzalez, L., Gomez-Rodriguez, G., LopezGarcia, J., Palma-Munoz, M., Trejo-Vazquez, I., Peralta-Higuera, A., Prado-Molina, J., Rodriguez-Aguilar, A., Mayorga-Saucedo, R., Gonzalez-Medrano, F., 2000, La condición actual de los recursos forestales en México: Resultados del Inventario Forestal Nacional 2000: Investigaciones Geográficas, 43, 183-203.

Running, S.W., Loveland, T.R., Pierce, L.L., Nemani, R.R., Hunt, E.R. Jr., 1995, A remote sensing based vegetation classification logic for global land cover analysis: Remote Sensing of Environment, 51, 39-48.

Sarle, W.S., 1994, Neural networks and statistical models (resumen), en Proceedings of the 19th Annual Special Air Service Group conference: Cary, Carolina del Norte, EUA, SAS Institute, 1538-1550.

Stehman, S.V., Czaplewski, R.L., 1998, Design and analysis for thematic map accuracy assessment: fundamental principles: Remote Sensing of Environment, 64, 331-344

Stone, T.A., Schlesinger, P., Houghton, R.A., Woodwell, G.M., 1994, A map of the vegetation of South America based on satellite imagery: Photogrammetric Engineering \& Remote Sensing, 60, 541-551.

Tucker, C.J., 1979, Red and Photographic Infrared Linear Combinations for Monitoring Vegetation: Remote Sensing of Environment, 8, 127-150.

Woodcock, C.E., Gopal S., 2000, Fuzzy set theory and thematic maps: accuracy assessment and area estimation: International Journal of Geographical Information Science, 14, 153-172.

Wulder, M.A., Franklin, S.E., White, J.C., Linke, J., Magnussen, S., 2006, An accuracy assessment framework for large-area land cover classification products derived from medium-resolution satellite data: International Journal of Remote Sensing, 27, 663-683.

Zhu, Z., Yang, L., Stehman, S.V., Czaplewski, R.L., 2000, Accuracy assessment for the U.S. Geological Survey regional land-cover mapping program: New York and New Jersey region: Photogrammetric Engineering \& Remote Sensing, 66, 1425-1435.

Recibido: Noviembre 17, 2009

Recibido corregido: Febrero 11, 2010

Aceptado: Marzo 03, 2010 